\title{
Prevalencia de sobrepeso y obesidad en estudiantes de la Escuela de Educación Básica "Dolores Sucre”, Azogues, Ecuador
}

\section{Overweight and obesity Prevalence of "Dolores Sucre" Elementary School students in Azogues, Ecuador}

\author{
Robert Iván Álvarez Ochoa ${ }^{1 *}$, Gabriela del Rosario Cordero Cordero ${ }^{1}$ y María Alicia Vásquez Calle Ma $^{2}$ \\ ${ }^{1}$ Universidad Católica de Cuenca \\ *rialvarezo@ucacue.edu.ec
}

DOI: https://doi.org/10.26871/killkana_salud.v4i1.579

\begin{abstract}
Resumen
El sobrepeso y la obesidad constituyen un problema de salud pública tanto en países desarrollados como en vías de desarrollo. Su presencia constituye un factor de riesgo para el desarrollo de enfermedades crónicas como la diabetes, hipertensión, entre otras en la vida adulta; de ahí la importancia de prevenirla, detectarla y tratarla a tiempo. El objetivo de la investigación fue determinar la prevalencia de sobrepeso y obesidad en estudiantes de la Escuela de Educación Básica "Dolores Sucre". Se efectuó una investigación descriptiva y transversal durante el año lectivo 2016-2017, se estudiaron 92 estudiantes de ocho y nueve años que aceptaron participar en el estudio. Se registraron edad, sexo, peso, talla, grasa corporal. Los principales resultados muestran que el total de estudiantes tuvieron sobrepeso $(21.7 \%)$ y obesidad $(10.9 \%)$. El porcentaje de grasa corporal fue más elevado en mujeres que en hombres en sus categorías alto y obesidad. Concluyendo, ambos géneros manifiestan sobrepeso, obesidad y valores altos de grasa corporal los mismos que componen factores de riesgo a largo plazo. Su detección temprana podría acarrear beneficios en la prevención de enfermedades cardiovasculares.
\end{abstract}

Palabras clave: Sobrepeso, obesidad, grasa corporal, estado nutricional, índice de masa corporal.

\begin{abstract}
Overweight and obesity constitute a public health problem in both developed and developing countries. Its presence is a risk factor for the development of chronic diseases such as diabetes and hypertension, among others in adulthood, hence the importance of prevention, detection and timely treatment. This research aimed to determine the overweight and obesity prevalence in students attending "Dolores Sucre" Elementary School. Descriptive and cross-sectional research analysis was conducted during the 2016-2017 school year. The participants were 92 students aged eight and nine years, who agreed to take part in the study. The participants' age, sex, weight, height, and body fat were documented. The main results show that all students presented overweight (21.7\%) and obesity (10.9\%). The body fat percentage was higher in women than in men, both in the "high" and "obesity" categories. In conclusion, both genders show overweight, obesity and highbody fat values, which are long-term risk factors. Early detection could bring benefits in the prevention of cardiovascular diseases.
\end{abstract}

Keywords: Overweight, obesity, body fat, school, nutritional status, body mass index.

\section{Introducción}

La obesidad es uno de los problemas de salud pública más serios en la actualidad, tal es así; que al año mueren alrededor de 2.8 millones de personas por esta causa. La población infantil no es la excepción, evidenciándose en el 2010, un incremento de la prevalencia de sobrepeso y obesidad del $4.2 \%$ al $6.7 \%$ en niños preescolares, lo que representa un aumento del $60 \%$ en los últimos veinte años. ${ }^{1}$

A nivel mundial, de acuerdo a estimaciones del International Obesity Task Force, de 30 a 45 millones de personas entre 5 y 17 años padece de obesidad, cifra que va en aumento en los últimos 20 años y se acentúa en los países desarrollados. ${ }^{2}$

En América Latina el sobrepeso y la obesidad han alcanzado cifras alarmantes evidenciándose en niños menores de 5 años una prevalencia de sobrepeso del $7.1 \%$, mientras que; en escolares el sobrepeso y obesidad estuvo presente en el $18.9 \%$ y $36.9 \%$ de educandos y en el $16.6 \%$ y $35.8 \%$ de adolescentes. ${ }^{3}$

En el Ecuador, el Fondo de las Naciones Unidas para la Infancia (UNICEF) 2014, registra un $8.6 \%$ de niños menores de 5 años con exceso de peso, porcentaje que se 
triplica entre los 5 y 11 años de edad $(29.9 \%)$ y en la población adolescente $(26 \%){ }^{4}$

El sobrepeso y la obesidad son considerados trastornos multifactoriales, los cuales están implicados en la predisposición y aparición de diversas patologías en la edad adulta como diabetes tipo 2, enfermedades cardiovasculares, dislipidemia, entre otras, que afectan el estado físico y psíquico del individuo, reduce la calidad de vida, y aumenta la mortalidad. El riesgo para desarrollar la enfermedad tiene múltiples fundamentos que van desde el cambio en la estructura familiar, la gran oferta de alimentos procesados, hábitos alimenticios inadecuados, sedentarismo, así como la herencia. ${ }^{5-8}$

Frente a esta problemática de salud pública a nivel mundial resulta importante conocer la realidad de nuestra comunidad para generar cambios encaminados a una mejor calidad de vida. Tomando en consideración lo expresado anteriormente, el presente estudio tuvo como objetivo determinar la prevalencia de sobrepeso y obesidad en estudiantes de la Escuela de Educación Básica "Dolores Sucre".

\section{Metodología}

Se realizó un estudio descriptivo transversal en 92 estudiantes de ocho y nueve años (34 masculinos y 58 femeninos pertenecientes a la escuela de educación básica "Dolores Sucre" durante el período lectivo 2016-2017. Se incluyó a los escolares de cuarto y quinto grado que se encontraban matriculados y cuyos padres o tutores dieron el permiso o consentimiento informado. Se investigó en un grupo etario lo más homogéneo posible con el fin de valorar la historia nutricional del mismo, poder realizar una comparación con mediciones hechas en el pasado en poblaciones parecidas y monitorear a futuro los cambios que pudieran ocurrir.

En base a datos del Ministerio de Educación de la provincia del Cañar (2014-2015) se estableció un universo poblacional de 180 estudiantes en las edades comprendidas en el estudio. A partir de dicho universo se calculó la muestra con el software EPIDAT 4.0, la que con un porcentaje de error del $5 \%$, un nivel de confianza del $95 \%$, una precisión absoluta entre 1 y $3 \%$ y un efecto de diseño de 1 se constituye en 92 estudiantes.

\subsection{Evaluación antropométrica}

Se capacitó a un grupo de 3 personas y se estandarizaron las mediciones de peso, talla y grasa corporal. Los participantes y colaboradores fueron reunidos en el dispensario médico de la institución una hora antes del recreo (09:00am). Cada colaborador midió en 2 ocasiones a cada niño/niña. La totalidad de los datos recogidos fueron registrados y revisados por un segundo observador.

Peso: se pesaron a los niños sin zapatos y con ropa ligera en una báscula/monitor Tanita ${ }^{\circledR}$ con capacidad de 300lb, graduación $0.21 \mathrm{~b}$ y precisión de $0.1 \mathrm{~kg}$.

Estatura: se midió a los niños con un estadímetro portátil marca Pelstar@ con precisión de $1 \mathrm{~mm}$.
Grasa corporal: se determinó utilizando un impedanciómetro bioeléctrico (BIA) marca Tanita ${ }^{\circledR}$ de 4 electrodos, con una precisión de porcentaje de grasa de $0.1 \%$. El equipo se calibró permanentemente y las mediciones se realizaron a temperatura ambiente $\left(18-24^{\circ} \mathrm{C}\right)$ considerando la técnica propuesta por diversos autores ${ }^{9-12}$ como por la sugerida por el manual de operaciones del instrumento y el protocolo de medición para bioimpedancia; según detallamos a continuación: no haber ingerido alimentos o líquidos previo a la medición, no haber realizado ejercicio intenso 12 horas antes, estar con la vejiga vacía y estar despojados de objetos metálicos.

Criterios de clasificación: se cotejaron las mediciones del porcentaje de grasa corporal por edad y género con las correspondientes curvas de referencia de grasa corporal para niños de McCarthy $2006 .^{13}$

De acuerdo al porcentaje de grasa corporal se establecieron los siguientes criterios por género y edad:

- $\quad$ Bajo en grasa: Niños (0\% a $12 \%)$, niñas (0\% a $14 \%)$.

- $\quad$ Saludable: Niños (13\% a $20 \%)$, niñas (15\% a $25 \%)$.

- Exceso de grasa: Niños (21\% a $25 \%)$, niñas (26\% a $30 \%)$.

- $\quad$ Obesidad: Niños (>26\%), niñas (>31\%).

Índice de masa corporal (IMC): se calculó mediante la fórmula: $\mathrm{IMC}=$ peso $(\mathrm{kg}) /$ estatura2 (m)

Criterios de clasificación: se cotejaron las mediciones de IMC por edad de cada niño/niña con las correspondientes a las curvas de crecimiento (CDC) del Ministerio de Salud Pública (MSP).

De acuerdo al puntaje $\mathrm{Z}$ de IMC-edad se establecieron las siguientes categorías:

- Delgadez $(<-2 \mathrm{DE})$

- Delgadez severa $(<-3 \mathrm{DE})$

- $\quad$ Normal (entre +1DE a -2DE)

- $\quad$ Sobrepeso ( $>+1 \mathrm{DE}$ a $+2 \mathrm{DE})$

- $\quad$ Obesidad $(>+2 \mathrm{DE}$ a $+3 \mathrm{DE})$

- Obesidad mórbida: ( $>+3 \mathrm{DE})$.

Los datos se analizaron en el software estadístico SPSS versión 21.0. El análisis estadístico descriptivo incluyó como medida de tendencia central la media y como medida de dispersión el desvío estándar. Se estimaron por género las prevalencias de sobrepeso y obesidad.

\subsection{Confiabilidad de las medidas}

Para establecer la calidad de las medidas antropométricas se recurrió a una doble medición a cada uno de los niños/niñas en todas las variables: peso, estatura, porcentaje de grasa corporal.

\section{Consideraciones éticas}

Este estudio se efectuó considerando los estándares de la Declaración de Helsinki sobre investigación en seres humanos. Posterior a la explicación de los objetivos del estudio, se solicitó el consentimiento a los padres o tutores y el asentimiento de los niños. 


\section{Resultados}

En la tabla 1 aparecen los datos de las características generales y antropométricas de la población de estudio expresadas en medias y desviación estándar por género.

La muestra estuvo conformada por 92 estudiantes (34 hombres y 58 mujeres) con edades de 8 y 9 años. Independiente de la edad, la media del peso, talla, IMC y grasa corporal en los varones fue menor que en las mujeres. La población evaluada al estratificarse por género presentó diferencias estadísticamente significativas en el peso, talla, IMC y porcentaje de grasa corporal $(\mathrm{p}<0,05)$. (Tabla 1$)$.

El IMC se clasificó de acuerdo a las CDC del MSP, donde un $23.9 \%$ de hombres presentaron normopeso y un $6.5 \%$ sobrepeso y obesidad respectivamente. En el género femenino del total de la muestra, el $43.5 \%$ se encontraron en normopeso, $15.2 \%$ en sobrepeso y $4.3 \%$ obesidad. (Tabla 2).

El porcentaje de grasa corporal obtenido por bioimpedancia eléctrica y estratificado en función del género evidencia un $10.9 \%$ de hombres que se encuentran en el nivel bajo en grasa, mientras que; un $13 \%$ de hombres y un $39.1 \%$ de mujeres se encontraron en el nivel saludable. Por su parte; en el nivel alto en grasa se localizaron un $8.7 \%$ de hombres como mujeres y en el nivel obesidad un $4.3 \%$ de hombres frente a un $15.2 \%$ de mujeres, evidenciando una sobrecarga ponderal sumando los valores de alto en grasa y obesidad de un $13 \%$ en el género masculino frente a un $23.9 \%$ en el género femenino. (Tabla 3 ).

Tabla 1. Media y desvío estándar de las variables antropométricas por género y grupos de edad

\begin{tabular}{lcccccccccc}
\hline Género & $\mathrm{N}$ & \multicolumn{2}{c}{ Peso $(\mathrm{kg})$} & \multicolumn{2}{c}{ Talla $(\mathrm{m})$} & \multicolumn{2}{c}{$\mathrm{IMC}\left(\mathrm{kg} / \mathrm{m}^{2}\right)$} & \multicolumn{3}{c}{$\mathrm{GC} \%$} \\
\cline { 2 - 10 } & & $\mathrm{X}$ & $\mathrm{DE}$ & $\mathrm{X}$ & $\mathrm{DE}$ & $\mathrm{X}$ & $\mathrm{DE}$ & $\mathrm{X}$ & $\mathrm{DE}$ \\
\hline Masculino & 34 & 27.70 & 6.39 & 122.45 & 14.29 & 16,9 & 3,06 & 20,07 & 8,63 \\
Femenino & 58 & 28.11 & 6.4 & 126.17 & 7.34 & 17,3 & 2,67 & 20,61 & 8,39 \\
\hline
\end{tabular}

IMC: Índice de Masa Corporal, GC: grasa corporal, X: media, D.E: desvío estándar

Tabla 2. Estratificación con base al IMC $\left(\mathrm{kg} / \mathrm{m}^{2}\right)$ en función del género

\begin{tabular}{lcccccc}
\hline Clasificación & \multicolumn{2}{c}{ Hombres $(\mathrm{n}=34)$} & \multicolumn{2}{c}{ Mujeres $(\mathrm{n}=58)$} & \multicolumn{2}{c}{ Total $(\mathrm{n}=92)$} \\
\cline { 2 - 7 } & $(\mathrm{Fi})$ & $(\%)$ & $(\mathrm{Fi})$ & $(\%)$ & $(\mathrm{Fi})$ & $(\%)$ \\
\hline Delgadez (<-2DE) & 0 & 0 & 0 & 0 & 0 & 0 \\
Delgadez severa (<-3DE) & 0 & 0 & 0 & 0 & 0 & 0 \\
Normopeso (entre +1DE a -2DE) & 22 & 23.9 & 40 & 43.5 & 62 & 67.4 \\
Sobrepeso >+1DE a +2DE & 6 & 6.5 & 14 & 15.2 & 20 & 21.7 \\
Obesidad (>+2DE a +3DE) & 6 & 6.5 & 4 & 4.3 & 10 & 10.9 \\
Obesidad mórbida (>+3DE) & 0 & 0 & 0 & 0 & 0 & 0 \\
\hline
\end{tabular}

Fi: Frecuencia absoluta (número de sujetos que se encuentran en cada una de las categorías); \%: porcentaje de la muestra

Tabla 3. Estratificación con base al porcentaje de grasa corporal en función del género

\begin{tabular}{|c|c|c|c|c|c|c|c|c|}
\hline \multirow[t]{2}{*}{ Clasificación } & \multicolumn{2}{|c|}{$\begin{array}{c}\text { Valores referenciales } \\
\% \text { Grasa corporal }\end{array}$} & \multicolumn{2}{|c|}{ Hombres $(n=34)$} & \multicolumn{2}{|c|}{ Mujeres $(n=58)$} & \multicolumn{2}{|c|}{ Total $(\mathrm{n}=92)$} \\
\hline & Hombres & Mujeres & $(\mathrm{Fi})$ & $(\%)$ & $(\mathrm{Fi})$ & $(\%)$ & (Fi) & $(\%)$ \\
\hline Bajo en Grasa & $0 \%$ a $12 \%$ & $0 \%$ a $14 \%$ & 10 & 10.9 & 0 & 0 & 10 & 10.9 \\
\hline Saludable & $13 \%$ a $20 \%$ & $15 \%$ a $25 \%$ & 12 & 13.0 & 36 & 39.1 & 48 & 52.2 \\
\hline Alto en grasa & $21 \%$ a $25 \%$ & $26 \%$ a $30 \%$ & 8 & 8.7 & 8 & 8.7 & 16 & 17.4 \\
\hline Obesidad & $>26 \%$ & $>31 \%$ & 4 & 4.3 & 14 & 15.2 & 18 & 19.6 \\
\hline
\end{tabular}

Fi: Frecuencia absoluta (número de sujetos que se encuentran en cada una de las categorías); \%: porcentaje de la muestra

\section{Discusión}

La prevalencia de la obesidad se ha incrementado en todo el mundo y es considerada por la OMS como la epidemia del siglo XXI. Se estima que el sobrepeso y obesidad en niños y adolescentes constituyen un factor de riesgo de padecer enfermedades no transmisibles como la diabetes, artrosis, cáncer y enfermedades cardiovasculares las cuales están relacionadas con problemas de obesidad en su edad de inicio y de duración. Por su parte; Serrano 14 afirma que la obesidad en el mundo es el quinto factor de riesgo y forma parte de las enfermedades más prevalentes tanto en la infancia como en la adolescencia.

La obesidad infantil tiene etapas de riesgo como son la prenatal, la infancia temprana, rebote adiposo entre los 5 a 7 años y adolescencia, a esto se suman otros elementos como 
la actividad física, sedentarismo, patrones nutricionales y factores familiares; dentro de los que destacan el estilo de crianza, peso de los padres, ambiente nutricional del hogar, ejemplo de los padres y por último componentes comunitarios tales como la etnia, estado socioeconómico, seguridad del barrio, programas escolares, acceso a instalaciones recreativas, entre otros. ${ }^{14}$

Escudero et al. ${ }^{15}$ son del criterio que la adiposidad visceral y la obesidad en los niños se encuentra asociado a un mayor riesgo cardiovascular y metabólico, independientemente del peso que llegue a tener el niño en la adultez. Por su parte, Peralta et al. ${ }^{16}$ mencionan que la obesidad se relaciona con diversos problemas de salud entre los que se encuentran colesterol alto, asma, trastornos del sueño, padecimientos hepáticos, diabetes mellitus tipo 2, enfermedades cardiovasculares y cáncer. Estos padecimientos que antes eran exclusivos de la edad adulta, ahora son cada vez más frecuentes en los infantes, tal es así que; niños obesos y con antecedentes familiares de hipertensión, presentan riesgo tres veces mayor de padecer presión arterial alta que niños normopesos. ${ }^{15,16}$

La BIA constituye una técnica aceptada y de bajo coste para determinar el porcentaje de grasa corporal tanto en adultos como en niños y tiene varios usos clínicos en el diagnóstico y seguimiento de pacientes con obesidad, sobrepeso, riesgo cardiovascular, anorexia, entre otros. ${ }^{17,18} \mathrm{El}$ porcentaje de grasa corporal compone un elemento básico en la exploración clínico inicial, de seguimiento y como un marcador temprano de sobrepeso y obesidad. ${ }^{19,20}$

En el presente estudio, la mitad de los investigados estuvo dentro del nivel saludable de grasa corporal, mientras que una tercera parte de los educandos $(37 \%)$ se encontraron en los niveles alto en grasa y obesidad, resultados que se corresponden con los encontrados por Hall López 22 donde 17 estudiantes de tercer y cuarto grado de primaria presentaron un porcentaje alto de grasa corporal de 35.9 \pm 5.5 .

De la misma manera; Ruiz Sánchez et al. ${ }^{21}$ encontraron valores similares de porcentaje de grasa corporal en estudiantes de dos escuelas primarias públicas de la ciudad de Durango, México, donde el promedio fue de 20.8 \pm 9.1 , hallazgo que coincide con el presente estudio (hombres: 20.07 \pm 8.63 , mujeres: $20.61 \pm 8.39$ ).

El IMC obtenido a través del peso y la talla del individuo es una medida muy útil para estimar el riesgo sobre la salud en la población. En la presente investigación se obtuvo un alto grado de sobrepeso $(21.7 \%)$ y obesidad $(10.9 \%)$. Estos valores son similares a los encontrados por Ruiz Sánchez et $a l .{ }^{21}$ en escolares entre 7 y 12 años donde el $35 \%$ presentó sobrepeso y obesidad en conjunto. Igualmente, en un grupo etario de 3 a 15 años, González et al. ${ }^{8}$ encontraron una prevalencia conjunta de sobrepeso y obesidad que superó el $25 \%$. Por el contrario; Sánchez et al. ${ }^{7}$ encontraron valores diferentes en escolares de 7 a 10 años, donde la prevalencia combinada de sobrepeso y obesidad fue de $5.1 \%$ y $10.2 \%$ respectivamente.
Resulta importante enfatizar que el IMC ha sido aceptado a nivel mundial en variados estudios epidemiológicos para este fin. $5,6,8,14,17,22$

\section{Conclusiones}

El sobrepeso y la obesidad tanto en niños como en adultos es considerado un problema de salud pública, razón por la cual es importante valorar la real extensión de esta epidemia y los factores que la condicionan, a fin de poder establecer medidas de intervención que contengan el aumento de su prevalencia y reduzcan la morbimortalidad que producen.

El porcentaje de grasa corporal y el IMC constituyen medidas antropométricas fáciles de aplicar y que brindan ayuda en la detección de problemas de sobrepeso y obesidad de forma temprana en la población escolar.

\section{Fuente de Financiamiento}

Este estudio es autofinanciado.

\section{Conflicto de Intereses}

No existen conflictos personales, profesionales, financieroso de otro tipo.

\section{Referencias Bibliográficas}

1. Ortiz-Pérez H, Molina-Frechero N, Martínez-Barbabosa I, Córdova-Moreno R. Contenido nutricional de los alimentos promovidos por el Canal 5 de la televisión mexicana dirigidos a la población infantil. Revista chilena de nutrición. 2015;42(3):260 - 266. Available from: https://scielo.conicyt.cl/scielo.php?script=sci_ arttext\&pid=S0717-75182015000300006\&nrm=iso.

2. Alba M, Martínez L, Pérez D. Prevención de la obesidad infantil como práctica de socialización. Revista Puertorriqueña De Psicología. 2017;28:336-352. Available from: http://dialnet.unirioja.es/servlet/articulo? codigo $=6234351$.

3. Rivera J, Cossío TD, Pedraza L, Aburto T, Sánchez T. Childhood and adolescent overweight and obesity in Latin America: a systematic review. Lancet Diabetes \& Endocrinology. 2014;2(4):321-332. Available from: http: //europepmc.org/abstract/med/24703050.

4. Ecuador U. UNICEF resaltó la necesidad de promover una alimentación saludable para combatir la obesidad y desnutrición infantil; 2014.

5. Teixeira Paiva AC, Campagnoli do Couto C, Pinheiro de Lemos Masson A, Aparecida Silveira Monteiro C, Fonseca Freitas C. Obesidade Infantil: análises antropométricas, bioquímicas, alimentares e estilo de vida. Revista Cuidarte. $2018 ; 9(3)$.

6. Wilches Reina G, Tarazona Martín J, Salazar Tapias JA, Lozano Portillo AJ, Rosas Galvis J. Factores de riesgo de sobrepeso y obesidad infantil en escolares de tres instituciones educativas de Cúcuta. INBIOM. 2017;4(1). 
7. Vicente Sánchez B, García K, González Hermida A, Saura Naranjo CE. Sobrepeso y obesidad en niños de 5 a 1217. años. Revista Finlay. 2017;7:47-53.

8. González Rosendo G, Villanueva Sánchez J, Alcantar Rodríguez VE, Quintero Gutiérrez AG. Sobrepeso y obesidad en niños y adolescentes de escuelas de tiempo completo de Morelos, México. Nutrición Hospitalaria. 2015;32:2588-2593.

9. Alvero-Cruz JR. Réplica: "variabilidad de la composición corporal medida por bioimpedanciometría eléctrica según condiciones de realización: influencia del ayuno y el reposo. Nutrición Hospitalaria. 2016;33:192-192.

10. Ramírez-Vélez R, Escobar-Cardozo GD, CorreaBautista JE, González-Jiménez E, Schmidt-RioValle J. Percentiles de grasa corporal por bioimpedancia eléctrica en niños y adolescentes de Bogotá, Colombia: estudio FUPRECOL. Archivos Argentinos De Pediatria. 2016;114. Available from: http://www.researchgate.net/ publication/284180031_Percentiles_de_grasa_corporal_ por_bioimpedancia_elctrica_en_nios_y_adolescentes_ de_Bogot_Colombia_estudio_FUPRECOL.

11. Orbegoso X, Alvarado A, Aballay L, Viola L. Importancia del reporte de metodología de Bioimpedancia Eléctrica (BIA) en estudios de composición corporal. Nutrición 2 Hospitalaria Organo Oficial De La So. 2015;32(2):959961. Available from: http://dialnet.unirioja.es/servlet/ articulo? codigo $=5227143$.

12. Leyva L, Ramentol C, Bethencourt J, Pestana E. Elementos teóricos y prácticos sobre la bioimpedancia eléc- 22 . trica en salud. Revista Archivo Médico de Camagüey. 2016;20(5). Available from: http://www.redalyc. org/articulo.oa?id=211147809014.

13. McCarthy HD, Cole TJ, Fry T, Jebb SA, Prentice AM. Body fat reference curves for children. International journal of obesity (2005). 2006;30(4):598-602. Available from: http://www.ncbi.nlm.nih.gov/sites/entrez?Db= pubmed $\&$ DbFrom $=$ pubmed $\&$ Cmd $=$ Link $\&$ LinkName $=$ pubmed_pubmed\&LinkReadableName $=$ Related $\%$ 20Articles\&IdsFromResult $=16570089$ \&ordinalpos $=$ 3\&itool=EntrezSystem2.PEntrez.Pubmed.Pubmed ResultsPanel.Pubmed_RVDocSum.

14. González M, Infantes-Paniagua 1, Martí I, Jordán O. Prevalencia de sobrepeso y obesidad infantil y su relación con factores sociodemográficos. Journal of Sport \& Health Research. 2018;10. Available from: http://www.researchgate.net/publication/325416359_ Prevalencia_de_sobrepeso_y_obesidad_infantil_y_su_ relacion_con_factores_sociodemograficos.

15. EscuderoLourdes GV, MoralesRomero LV, ValverdeOcaña C. Riesgo cardiovascular en población infantil de 6 a 15 años con obesidad exógena. Rev Med Inst Mex Seg Soc. 2014;52(1). Available from: http://www.redalyc.org/ articulo.oa?id=457745485010.

16. Peralta J, Gómez J, Estrada B, Karam R, Cruz M. Genética de la obesidad infantil. Rev Méd Inst Mex Seg Soc.
2014;52(1).

18. Espinosa Reyes TM, Hernández Moreno JA, Hernández M, Domínguez Alonso E. Composición corporal y factores relacionados en pacientes con hiperplasia adrenal congénita. Revista Cubana de Endocrinología. 2018;29:114.

19. Duno M, Barón MA, Solano L. Determinación de porcentaje de grasa corporal a través del método de dilución isotópicacon deuterio en niños entre 6 a 11 años de edad. Naguanagua, Estado Carabobo. Salus. 2018;22(1):26-31.

20. Pinheiro ACDB, Filho NS, França AKTDC, Fontenele AMM, Santos AMD. Sensitivity and specificity of the body mass index in the diagnosis of obesity in patients with non-dialysis chronic kidney disease: a comparison between gold standard methods and the cut-off value purpose. Nutricion hospitalaria. 2019;36(1):73-79. Available from: http://www.ncbi.nlm.nih.gov/sites/ entrez?Db=pubmed $\&$ DbFrom $=$ pubmed $\&$ Cmd $=$ Link $\&$ LinkName=pubmed_pubmed\&LinkReadableName $=$ Related\%20Articles\&IdsFromResult $=30834758 \&$ ordinalpos=3\&itool=EntrezSystem2.PEntrez.Pubmed. Pubmed_ResultsPanel.Pubmed_RVDocSum.

. Ruiz-Sánchez E, Bañuelos-Barrera Y, Bañuelos-Barrera P, Álvarez Aguirre A, Valles-Verdín M, Domínguez-Chávez C. Porcentaje de grasa corporal en escolares y su asociación con el estilo de vida y macronutrientes. Revista Cuidarte. 2015;6(2).

. Vaquero-Álvarez M, Romero-Saldaña M, Valle-Alonso J, Llorente Cantarero FJ, Blancas-Sánchez IM, Fonseca Del Pozo FJ. Study of obesity in a rural children population and its relationship with anthropometric variables. Atencion primaria. 2019;51(6):341-349. Available from: http://www.ncbi.nlm.nih.gov/sites/ entrez? $\mathrm{Db}=$ pubmed $\& \mathrm{DbFrom}=$ pubmed $\&$ Cmd $=$ Link $\&$ LinkName=pubmed_pubmed $\&$ LinkReadableName $=$ Related\%20Articles\&IdsFromResult=29789165\& ordinalpos $=3 \&$ itool=EntrezSystem2.PEntrez.Pubmed. Pubmed_ResultsPanel.Pubmed_RVDocSum.

Recibido: 10 de noviembre de 2019

Aceptado: 16 de diciembre de 2019 
